\title{
Microscopic diffusion of partly ionized metals in the Sun and metal-poor stars
}

\begin{abstract}
H. Schlattl ${ }^{\star}$
Astrophysics Research Institute, Liverpool John Moores University, Twelve Quays House, Egerton Wharf, Birkenhead CH41 1LD, UK

Received 16 April 2002 / Accepted 21 August 2002

Abstract. An improved microscopic diffusion in stars is presented considering in detail the partly ionized stages of metals. Besides, the influence of degenerate electron-gas and of the contribution of radiation to the total pressure has been accounted for. The solution of the diffusion equations is then performed following the scheme of Thoul et al. (1994). By defining one mean charged ion per element very few modifications are necessary to solve the improved diffusion scheme. (A portable FORTRAN routine is provided.) The change in the sound-speed profile of a solar model obtained with the new diffusion description is at most about $25 \%$ at $r=0.6 R_{\odot}$. The biggest effect on low-mass stars is expected near the turn off, where the convective envelope is shallowest. However, only a difference of at most $40 \mathrm{~K}$ in the effective temperature could be observed when assuming either fully or partly ionized metals in the diffusion equation. Nevertheless, the surface metal distribution is strongly altered.
\end{abstract}

Key words. Sun: interior - stars: evolution - stars: abundances - Hertzsprung-Russel (HR) diagram

\section{Introduction}

In the last few years the precise measurement of solar oscillations has challenged theorists to compute solar models of gradually higher accuracy. This demanded an improvement of the existing input physics like equation of state (Rogers et al. 1996), opacities (Iglesias \& Rogers 1996) and nuclear reaction rates (Adelberger et al. 1998). In addition, the formerly neglected process of microscopic diffusion has been found to improve the agreement of solar models with helioseismic data considerably (Bahcall \& Pinsonneault 1992). Nevertheless, there exists a significant discrepancy in the sound speed just below the convective between the theoretical predicted and the helioseismic determined value, the reason of which is still unknown.

Unfortunately, the expression "diffusion" is not always used in the literature to cover the same physical process(es). In order to avoid confusion, "diffusion" is defined in this work like, e.g., in Bahcall et al. (1998) or Salaris et al. (2000), to describe abundance changes due to pressure, temperature, and concentration gradients neglecting the effects of radiative forces.

Beside solar models diffusion is now also implemented in many stellar calculations, e.g., for the computation of globular cluster isochrones (Castellani et al. 1997; Salaris et al. 2000). With the improved models globular clusters have been found to be about 1 Gyr younger than determined previously by models without diffusion.

\footnotetext{
* e-mail: hs@astro.livjm.ac.uk
}

However, recent observations of the surface iron abundance of near turn-off stars (Ramírez et al. 2001; Ramírez \& Cohen 2002) suggests that diffusion is much less efficient in metalpoor stars with thin convective envelopes than theoretically predicted. Even worse, the models predict an almost total depletion of heavy elements in the surface of such stars at the turn off (see Sect. 3.2). Including the here neglected effect of radiative forces, the opposite effect can be obtained for some elements, e.g., $\mathrm{Fe}$ may then be enhanced considerably at the surface (Richard et al. 2002). Thus, radiative levitation is important in certain evolutionary phases, and should be included in future models.

Nevertheless, by assuming an additional mixing process below the convective envelope it would be possible to inhibit any diffusion process. Such a process is also favoured to reduce the discrepancy in the sound speed just below the solar convective zone (Richard et al. 1996).

Various mechanisms have been proposed to cause additional mixing. Before, however, being able to determine the extent of additional mixing processes or other non-standard physics, diffusion should be followed accurately. Therefore, the validity of physical assumptions used to compute the diffusion efficiency is investigated in this work. A common assumption in the calculation of diffusion constants is the complete ionization of all elements. Basically in all stellar model computations including diffusion (e.g. Castellani et al. 1997; Salaris et al. 2000; Weiss \& Schlattl 2000; Chaboyer et al. 2001) this approximation is made. Exception are the models of Richer et al. (2000) or Richard et al. (2002) which account for partial ionization and radiative levitation in the diffusion treatment. 
Since the cross section for the main scattering process of ions in the stellar plasma, the Coulomb scattering, is proportional to the square of the ion charge, deviations from complete ionization may have an important influence on the diffusivity of the elements. But the diffusion constant of a specific element is not simply direct proportional to the cross section, because diffusion has to obey the laws of mass and charge conservation. Thus, the cross section of each element has to be considered in relation to the ones of all other elements. An exact knowledge of the ionization stage of each element is therefore necessary.

In order to obtain more accurate microscopic diffusion constants the assumption of fully ionized metals is dropped in this work. Instead, the ionization stage of each metal is considered in detail, where the ionization degrees of each element are determined by using an up-to-date EOS of Irwin (2000).

In the next section the implementation of partly ionized elements into the solution of Burgers' equations (Burgers 1969) is described. The changes in the solar sound-speed profile and in the evolution of metal-poor stars using this improved description are discussed afterwards.

\section{Partly ionized elements in Burgers' equation}

The method to calculate diffusion constants as described by Thoul et al. (1994), denoted TBL hereafter, is followed, where the basic concepts and quantities can be found. The aim is to improve this method in particular by including partly ionized atoms. In addition, a possible non-ideal electron gas and the contribution of radiation pressure to the hydrostaticequilibrium equation are taken into account ${ }^{1}$, the effects of which are neither considered by TBL. Besides, in this section nuclear burning is neglected; it can simply be added afterwards $^{2}$.

\subsection{Basic equations}

An overall neutral plasma consisting of $S$ species is assumed, where a species is defined to be an element in one particular ionization stage. Electrons are supposed to be species No. $S$, and are usually not mentioned explicitly. Burgers' equation for mass conservation can then be written as (cf. Eq. (11) in TBL)

$\frac{\partial n_{\mathrm{s}}}{\partial t}=-\frac{1}{r^{2}} \frac{\partial}{\partial r}\left(r^{2} \omega_{\mathrm{s}} n_{\mathrm{s}}\right)$,

where $r$ is the radial distance from the centre, and $n_{\mathrm{s}}$ is the number density of ion $\mathrm{s}$. The diffusion velocity $\omega_{\mathrm{s}}$ has to be determined by solving the momentum- and energy-conservation equations (cf. Eqs. (18.1) and (18.2) of Burgers (1969), neglecting magnetic fields)

$\frac{\mathrm{d} P_{\mathrm{s}}}{\mathrm{d} r}+\frac{\rho_{\mathrm{s}}}{\rho} \frac{\mathrm{d} P_{\mathrm{gas}}}{\mathrm{d} r}-\rho_{\mathrm{es}} E=f_{1}\left(\mathrm{~K}, \boldsymbol{m}, \omega, \boldsymbol{r}_{\mathrm{h}}\right)$

${ }^{1}$ A FORTRAN77 routine containing all the non-standard effects in the computation of the diffusion constants is publicly accessible under http://www. astro.livjm.ac.uk/ ${ }^{\sim}$ hs

${ }^{2}$ In the models presented nuclear burning is, of course, fully included. and

$\frac{5}{2} P_{\mathrm{s}} \frac{\mathrm{d} \ln T}{\mathrm{~d} r}=f_{2}\left(\mathrm{~K}, \boldsymbol{m}, \omega, \boldsymbol{r}_{\mathrm{h}}\right)$,

where $P_{\mathrm{s}}, \rho_{\mathrm{s}}\left(=n_{\mathrm{s}} m_{\mathrm{s}}\right)$, and $\rho_{\mathrm{es}}\left(=n_{\mathrm{s}} q_{\mathrm{s}}\right)$ are the partial pressure, mass density, and charge density, respectively, for ion s with $m_{\mathrm{s}}$ being its mass and $q_{\mathrm{s}}$ its charge; $E$ is the electric field strength. The vectors $\boldsymbol{m}$ and $\boldsymbol{\omega}$ contain the mass and diffusion velocity for each ion s. For a detailed definition of the friction-coefficient tensor $\mathrm{K}$, the residual heat-flow vector $\boldsymbol{r}_{\mathrm{h}}$, and the functions $f_{1}$ and $f_{2}$ the reader is referred to TBL.

The total pressure $P$ is composed of radiation $\left(P_{\text {rad }}\right)$ and gas pressure $\left(P_{\text {gas }}\right)$, thus

$P=P_{\mathrm{rad}}+P_{\mathrm{gas}}=P_{\mathrm{rad}}+\sum P_{\mathrm{s}}=\sum \frac{1}{\beta} P_{\mathrm{s}}$,

where the standard definition $\beta=P_{\text {gas }} / P$ has been employed. Since the departure from local charge neutrality is very small (cf. TBL), $\rho_{\mathrm{e}} E$ is close to zero, and thus the usual hydrostaticequilibrium equation

$\frac{\mathrm{d} P}{\mathrm{~d} r}=-g \rho$

can be used, with $g$ being the gravitational acceleration. Hence, Eq. (2) becomes

$\frac{\mathrm{d} P_{\mathrm{s}}}{\mathrm{d} r}+\beta g \rho_{\mathrm{s}}-\frac{\rho_{\mathrm{s}}}{\rho} \frac{\mathrm{d} \beta}{\mathrm{d} r}-\rho_{\mathrm{es}} E=f_{1}\left(\mathrm{~K}, \boldsymbol{m}, \boldsymbol{\omega}, \boldsymbol{r}_{\mathrm{h}}\right)$.

By two additional constraints, current neutrality,

$\sum q_{\mathrm{s}} n_{\mathrm{s}} \omega_{\mathrm{s}}=0$

and local mass conservation,

$\sum m_{\mathrm{s}} n_{\mathrm{s}} \omega_{\mathrm{s}}=0$,

Eqs. (3) and (5) are completed to form a closed set of $2 S+2$ equations for the unknown quantities $\omega, \boldsymbol{r}_{\mathrm{h}}, g$ and $E$.

For a large number of species with varying abundances these equations are preferentially solved numerically. For this purpose, they are reformulated to be more suited for stellar evolution calculations.

The partial pressure $P_{\mathrm{s}}$ for atom $\mathrm{s}$ is given by the ideal gas equation

$P_{\mathrm{s}}=n_{\mathrm{s}} k_{\mathrm{B}} T$.

The electron pressure in stellar envelopes is well described by this formula, too. However, in the deep radiative regions of low-mass stars, and in particular in white dwarfs, the electron gas is degenerate. Therefore, an "effective" electron density is introduced

$\tilde{n}_{\mathrm{e}}=\frac{P_{\mathrm{e}}}{k_{\mathrm{B}} T}$

such that the total pressure can be written

$P=\frac{1}{\beta} \sum n_{\mathrm{s}} k_{\mathrm{B}} T$, 
where $n_{\mathrm{S}}=\tilde{n}_{\mathrm{e}}$. Furthermore the concentration $C_{\mathrm{S}}$ is defined by

$C_{\mathrm{s}}=\frac{n_{\mathrm{s}}}{\tilde{n}_{\mathrm{e}}}$

analogously to TBL.

With $C=\sum C_{\mathrm{s}}$ one can now write

$\frac{P_{\mathrm{s}}}{\beta P}=\frac{P_{\mathrm{s}}}{\sum P_{\mathrm{s}}}=\frac{n_{\mathrm{s}}}{\sum n_{\mathrm{s}}}=\frac{C_{\mathrm{s}}}{C}$,

and hence the first term of Eq. (5) becomes

$\frac{\mathrm{d} P_{\mathrm{s}}}{\mathrm{d} r}=\frac{\mathrm{d} \frac{C_{\mathrm{s}}}{C} \beta P}{\mathrm{~d} r}=\frac{C_{\mathrm{s}}}{C} \beta P\left(\frac{\mathrm{d} \ln \beta P}{\mathrm{~d} r}+\frac{\mathrm{d} \ln \left(C_{\mathrm{s}} / C\right)}{\mathrm{d} r}\right)$.

Taking into consideration that in TBL the pressure $P$ has been defined to be solely the gas pressure, this term seems to agree with TBL. However, here $C_{\mathrm{s}}$ has been defined via Eq. (10) instead of TBL's Eq. (22) $\left(C_{\mathrm{s}}=n_{\mathrm{s}} / n_{\mathrm{e}}\right)$, which accounts for a possible degeneracy of the electron gas.

Demanding charge neutrality, $\sum_{\mathrm{s} \neq \mathrm{e}} q_{\mathrm{s}} n_{\mathrm{s}}=-q_{\mathrm{e}} n_{\mathrm{e}}$, one gets rid of the dependence on one species, e.g. $\mathrm{He}^{++}$. Thus,

$\zeta_{\mathrm{He}^{++}} C_{\mathrm{He}^{++}}=\left(\tilde{C}_{\mathrm{e}}-\sum_{\mathrm{s} \neq \mathrm{He}^{++}, \mathrm{e}} \zeta_{\mathrm{s}} C_{\mathrm{s}}\right)$,

where $\zeta_{\mathrm{s}}$ is the ionization stage of ion $\mathrm{s}\left(\zeta_{\mathrm{s}}=0\right.$ for neutral atoms) and

$\tilde{C}_{\mathrm{e}}=\frac{n_{\mathrm{e}}}{\tilde{n}_{\mathrm{e}}}$

Similarly, the 1.h.s. of Eq. (3) becomes

$\frac{5}{2} P_{\mathrm{s}} \frac{\mathrm{d} \ln T}{\mathrm{~d} r}=\frac{5}{2} \frac{C_{\mathrm{s}}}{C} \beta P \frac{\mathrm{d} \ln T}{\mathrm{~d} r}$.

Setting Eqs. (8)-(15) into Eqs. (3)-(7) one finds, after some algebraic manipulations, that the diffusion velocity can be written in general as (see Appendix A)

$\omega_{\mathrm{s}}=\frac{T^{5 / 2}}{\rho} \xi_{\mathrm{s}}$,

where

$$
\begin{aligned}
\xi_{\mathrm{s}}= & A_{\mathrm{s}}^{\mathrm{P}} \frac{\mathrm{d} \ln P}{\mathrm{~d} r}+A_{\mathrm{s}}^{\mathrm{T}} \frac{\mathrm{d} \ln T}{\mathrm{~d} r}+\sum_{t \neq e, \mathrm{He}^{++}} A_{\mathrm{t}, \mathrm{s}}^{\mathrm{C}} \frac{\mathrm{d} \ln C_{\mathrm{t}}}{\mathrm{d} r} \\
& +A_{\mathrm{s}}^{\beta} \frac{\mathrm{d} \ln \beta}{\mathrm{d} r}+A_{\mathrm{e}, \mathrm{s}}^{\mathrm{C}} \frac{\mathrm{d} \ln \tilde{C}_{\mathrm{e}}}{\mathrm{d} r}+\sum_{t \neq e} A_{\mathrm{t}, \mathrm{s}}^{\zeta} \frac{\mathrm{d} \ln \zeta_{\mathrm{t}}}{\mathrm{d} r} .
\end{aligned}
$$

The diffusion coefficients $A^{\mathrm{P}}, A^{\mathrm{T}}, A^{\mathrm{C}}, A^{\beta}$, and $A^{\zeta}$ are obtained by the solution of Eqs. (3)-(7). For convenience, the six terms of Eq. (17) are denoted $\xi_{\mathrm{s}}^{\mathrm{P}}, \xi_{\mathrm{s}}^{\mathrm{T}}, \xi_{\mathrm{s}}^{\mathrm{C}}, \xi_{\mathrm{s}}^{\beta}, \xi_{\mathrm{s}}^{\mathrm{e}}$, and $\xi_{\mathrm{s}}^{\zeta}$, respectively. Compared to the analogous equation in TBL (Eq. (40)), three additional terms appear here. They should not be considered as new types of diffusion but rather as corrections to pressure (term 4) and concentration diffusion (terms 5 and 6).

In particular, the fourth term in Eq. (17) $\left(\xi_{\mathrm{s}}^{\beta}\right)$ corrects for the appearance of a radiation pressure in the hydrostaticequilibrium equation. The fifth term $\left(\xi_{\mathrm{s}}^{\mathrm{e}}\right)$ takes into account that for degenerate electrons the ideal linear relation between pressure and density is not valid any more (Eq. (14)). Thus, this

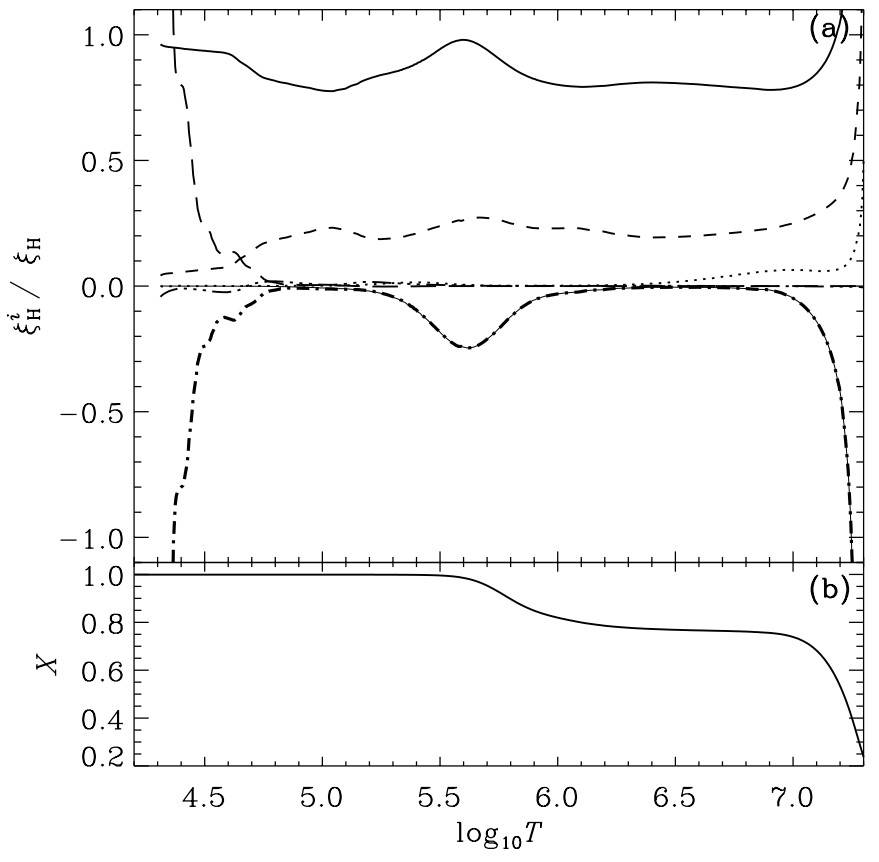

Fig. 1. a) The contributions of the six terms in Eq. (17) to the diffusion velocity of hydrogen in a $1.1 M_{\odot}$ star near the TO with initial $[\mathrm{Fe} / \mathrm{H}]=-1.3: \xi_{\mathrm{s}}^{\mathrm{P}}$ (solid line), $\xi_{\mathrm{s}}^{\mathrm{T}}$ (short-dashed), $\xi_{\mathrm{s}}^{\mathrm{C}}$ (dash-dotted), $10 \times \xi_{\mathrm{s}}^{\beta}$ (dash-dot-dot-dotted), $10 \times \xi_{\mathrm{s}}^{\mathrm{e}}$ (dotted), and $\xi_{\mathrm{s}}^{\zeta}$ (long-dashed). The thin solid line represents $\xi_{\mathrm{s}}^{\mathrm{C}}+\xi_{\mathrm{s}}^{\zeta}$. b) The hydrogen mass fraction in this model.

term is only important, when strong gradients in the electron degeneracy appear (e.g. in white dwarfs).

In Fig. 1a the contribution of each of the 6 terms in Eq. (17) to the diffusion velocity $\omega$ of $\mathrm{H}$ in a $1.1 M_{\odot}$ star near the turn off (TO) are shown. Pressure $\left(\xi_{\mathrm{s}}^{\mathrm{P}}\right)$ and temperature diffusion $\left(\xi_{\mathrm{s}}^{\mathrm{T}}\right)$ are clearly dominating in most parts of the stellar interior. Near the centre, where already most of the $\mathrm{H}$ has been consumed, concentration diffusion $\left(\xi_{\mathrm{s}}^{\mathrm{C}}\right)$ becomes important. The contribution of $\xi_{\mathrm{s}}^{\beta}$ remains very small throughout the star, while $\xi_{\mathrm{s}}^{\mathrm{e}}$ increases toward the centre, where the electron degeneracy is gradually increasing.

The last term in Eq. (17) $\left(\xi_{\mathrm{s}}^{\zeta}\right)$, which originates from the demand for charge neutrality (Eq. (13)), needs a more thorough consideration. It would disappear, if each ion is treated as a separate species, because the ionization stage $\zeta$ of each species, i.e., the number of electrons per ion, is constant. Charge neutrality is then, as in the case of complete ionization, automatically conserved by the definition of $A^{\mathrm{C}}$. But if, as shown below, a "mean" ion per element is defined which is in an average ionization stage $\bar{\zeta}$, the number of electrons per species is varying in the ionizing regions of a star. This would cause artificial concentration diffusion. Due to $\xi_{\mathrm{s}}^{\zeta}$ this effect is compensated, ensuring that only the total number density of a certain element is relevant for its concentration diffusion, independent of the respective ionization stages in two neighbouring layers.

Note that using one mean charged ion per element cannot lead to ambipolar diffusion (see Babel \& Michaud 1991), and references therein). Hence the ionization state of each element is determined in this case solely by the local thermodynamic equilibrium and cannot be modified by diffusion. 


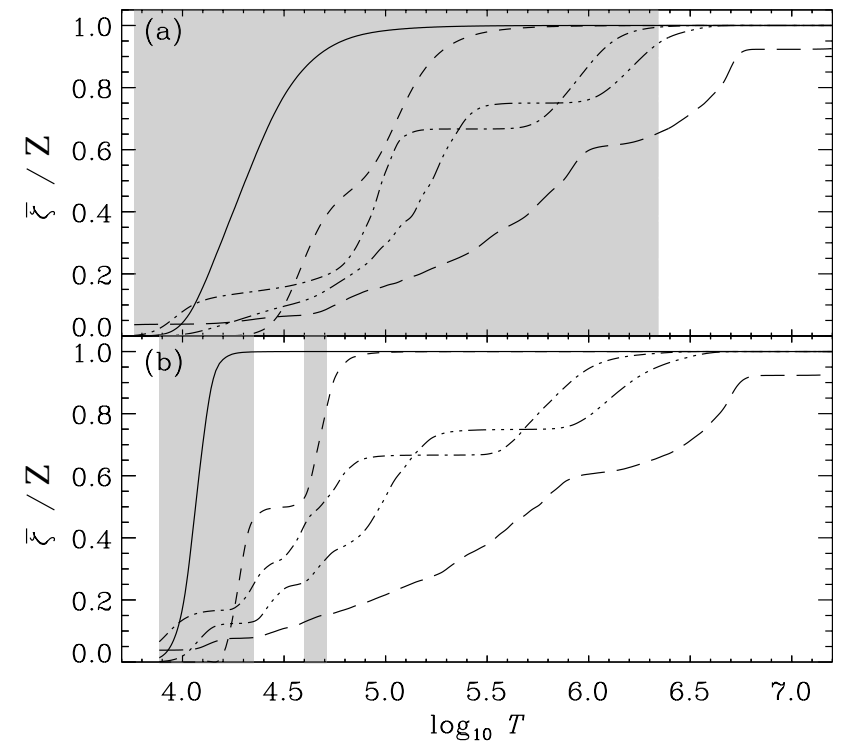

Fig. 2. a) The mean ionization degrees $\bar{\zeta}$ of $H$ (solid line), He (short dashed), C (dash-dotted), O (dash-dot-dot-dotted), and Fe (long dashed) in the Sun, normalized to their respective charge number $\mathcal{Z}$ (obtained from Irwin's 2000 EOS). The envelope convective zone is located within the grey shaded area. b) Same as a) but for a $1.1 M_{\odot}$ star near the TO with $[\mathrm{Fe} / \mathrm{H}]=-1.3$.

An example is provided in Fig. 1a, where mean charged ions per element were used to compute the contributions to the diffusion velocity. In the layers near $\log _{10} T \approx 4.4$, just below the convective envelope (cf. Fig. 2), $\mathrm{H}$ is not completely ionized $\left(\zeta_{\mathrm{H}} \approx 0.995\right)$. This small deviation from complete ionization causes a large negative contribution of the concentration diffusion term (dash-dotted line) to $\omega_{\mathrm{H}}$, although the hydrogen abundance is constant in this region (Fig. 1b). However, by the last term in Eq. (17) this artificial concentration diffusion is counterbalanced such that the sum of $\xi_{\mathrm{s}}^{\mathrm{C}}$ and $\xi_{\mathrm{s}}^{\zeta}$ remains zero in this area (thin solid line in Fig. 1a).

The diffusion of ions into regions, where they are out of thermodynamic equilibrium, yields an additional energy sink (or source). However, the ionization of the most abundant elements, which are usually hydrogen and helium, leads in most relevant cases to the development of convective zones (Fig. 2), where mixing occurs on much shorter timescales than diffusion. Thus, in the radiative layers only less abundant elements may be in differently ionized stages. The out-of-equilibrium distribution of their ions leads only to a small amount of energy compared to the total internal energy of the plasma, and is thus neglected here.

\subsection{Linear approximation for $\omega_{s}$}

Using a mean ionization stage for all elements, instead of treating each ion separately, is valid if the diffusion velocity $\omega_{\mathrm{s}}^{\mathrm{i}}$ is a linear function of the charge of ion i of element s. Then

$\omega_{\mathrm{s}}=\omega_{\mathrm{s}}(\zeta)=\omega_{\mathrm{s}, 0}+\zeta \delta \omega_{\mathrm{s}}$

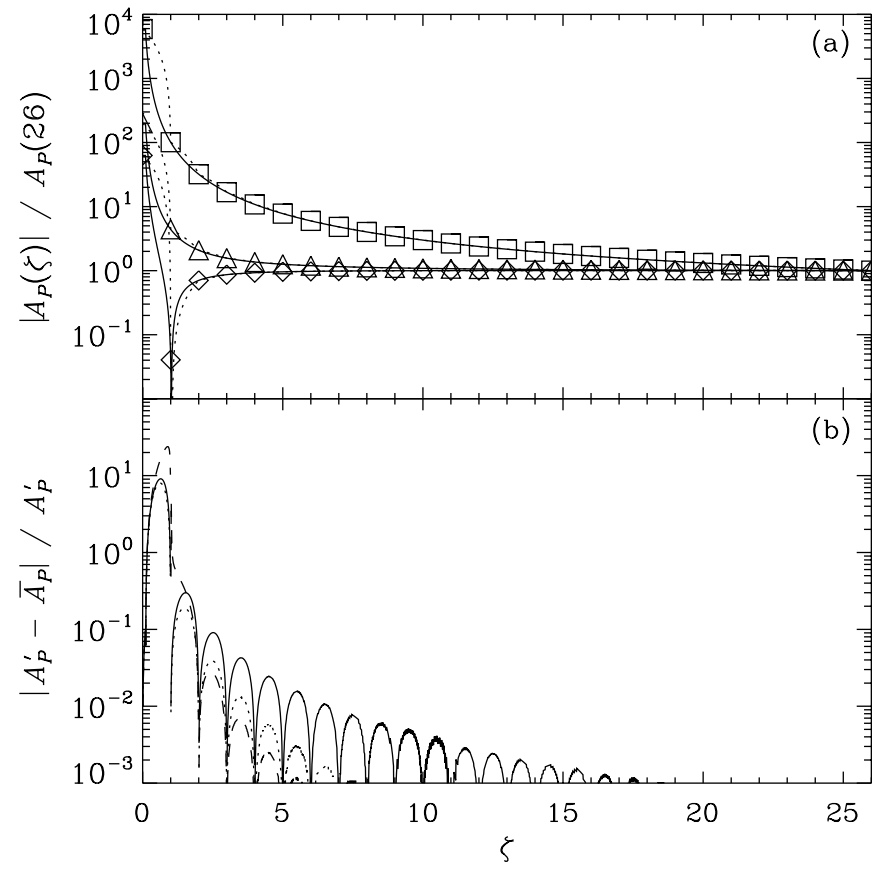

Fig. 3. a) Dependence of the pressure diffusion constant $A_{\mathrm{P}}$ of $\mathrm{H}(\triangle)$, $\mathrm{He}(\diamond)$ and $\mathrm{Fe}(\square)$ on the charge $(\zeta)$ of the Fe-ion in a mixture of $X=0.76, Y=0.23$ and $X(\mathrm{Fe})=0.01$ at $T=2.24 \times 10^{6} \mathrm{~K}$ and $\rho=$ $0.19 \mathrm{~g} / \mathrm{cm}^{3}$. Using a mixture of two different Fe-ions with charges $\zeta(i)$ and $\zeta(i+1)$ the dotted line is obtained for the mean diffusion constants $\bar{A}_{\mathrm{P}}$ ("exact value"), while the solid line represents the result employing the mean charge $\bar{\zeta}$ ("linear approximation"). At $\zeta \approx 1 A_{\mathrm{P}}^{\prime}(\mathrm{He})$ changes its sign. b) The relative difference between $\bar{A}_{\mathrm{P}}$ and $A_{\mathrm{P}}^{\prime}$ for $\mathrm{H}$ (dotted line), $\mathrm{He}$ (dashed) and $\mathrm{Fe}$ (solid).

and therefore

$\sum_{\mathrm{i}} \omega_{\mathrm{s}}^{\mathrm{i}} n_{\mathrm{s}}^{\mathrm{i}}=\sum_{\mathrm{i}}\left(\omega_{\mathrm{s}, 0}+\zeta_{\mathrm{s}}^{\mathrm{i}} \delta \omega_{\mathrm{s}}\right) n_{\mathrm{s}}^{\mathrm{i}}=\left(\omega_{\mathrm{s}, 0}+\bar{\zeta}_{\mathrm{s}} \delta \omega_{\mathrm{s}}\right) n_{\mathrm{s}}$

is obtained, where $\zeta_{\mathrm{s}}^{\mathrm{i}}$ are the discrete ionization stages of $\mathrm{s}$, and $\overline{\zeta_{\mathrm{s}}}$ is the mean charge defined by $\overline{\zeta_{\mathrm{s}}}=\left(\sum_{\mathrm{i}} \zeta_{\mathrm{s}}^{\mathrm{i}} n_{\mathrm{s}}^{\mathrm{i}}\right) / n_{\mathrm{s}}$ with $n_{\mathrm{s}}=\sum_{\mathrm{i}} n_{\mathrm{s}}^{\mathrm{i}}$.

In order to verify to what extent the assumption of $\omega_{\mathrm{s}}$ being linear in $\zeta$ is justified, the diffusion coefficients for a simple mixture of hydrogen, helium and iron with $X=0.76, Y=0.23$ and $X(\mathrm{Fe})=0.01$ have been computed. Conditions similar to those at the base of the solar convective envelope were chosen, i.e., $T=2.24 \times 10^{6} \mathrm{~K}$ and $\rho=0.19 \mathrm{~g} / \mathrm{cm}^{3}$.

In Fig. 3a the symbols mark the pressure diffusion coefficients $A_{\mathrm{P}}$ of $\mathrm{H}, \mathrm{He}$, and $\mathrm{Fe}$ for different ionization levels of the latter, while $\mathrm{H}$ and $\mathrm{He}$ are assumed to be fully ionized. For Fe I the interactions can no longer be described by Coulomb scattering, but are due to the atomic polarisability (see e.g. Michaud et al. 1978). It has been found that the diffusion coefficients of a neutral atom is about a factor of 100 higher than of its single charged ion (see Gonzalez et al. 1995). To obtain a similar value but using the Coulomb scattering description an "effective" ionization degree of 0.1 for neutral atoms is adopted. This certainly only yields approximate values for the diffusion constants, and should be improved in future works. 


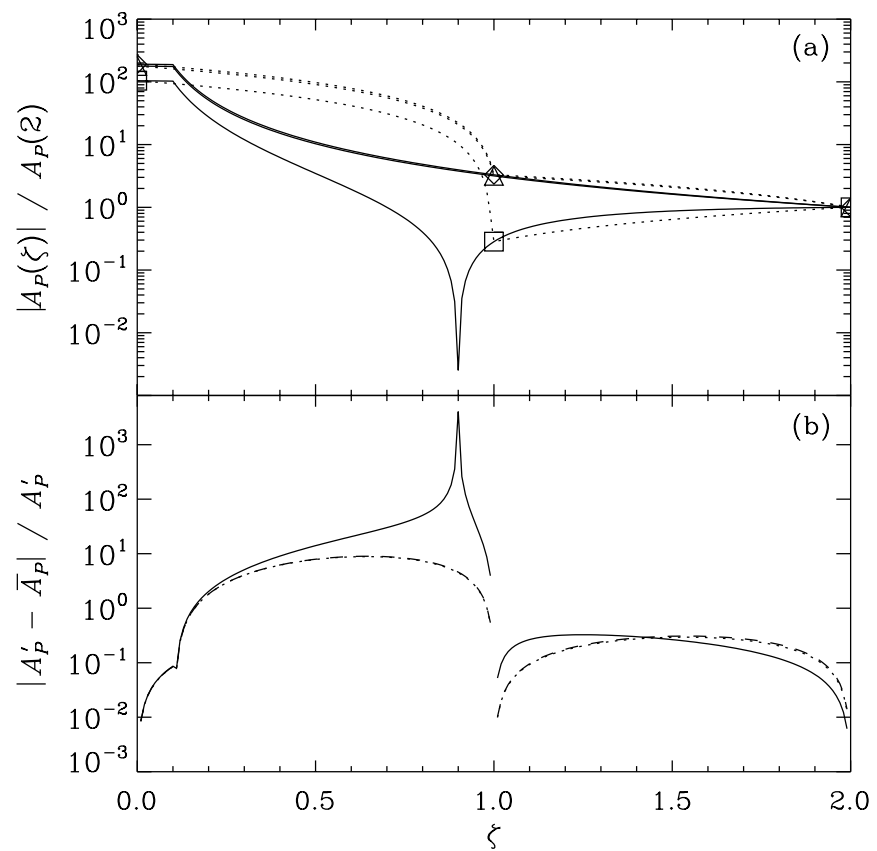

Fig. 4. Same as Fig. 3 but for varying He-ionization degrees. Note that at $\zeta \approx 0.9 A^{\prime}(\mathrm{Fe})$ changes the sign.

Obviously, $A_{\mathrm{P}}$ is not a simple linear function of $\zeta$, but with increasing ionization the non-linearity becomes smaller. The dotted line in Fig. 3a shows the mean diffusion coefficient $\bar{A}_{\mathrm{P}}$ for a mixture consisting of two Fe-ions with number density $n_{i}$ and $n_{i+1}$ and discrete charges $\zeta_{i}$ and $\zeta_{i+1}$, respectively. If instead the mean ionization stage $\bar{\zeta}=\frac{n_{i} \zeta(i)+n_{i+1} \zeta(i+1)}{n_{i}+n_{i+1}}$ is used, $A_{\mathrm{P}}^{\prime}(\zeta)$ follows the solid line.

In Fig. $3 b$ the relative error between the two values is plotted. Because of the large mean free path of Fe I, $A_{\mathrm{P}}(\mathrm{He})$ has a different sign for neutral than for ionized Fe causing He to diffuse in the same direction as $\mathrm{H}$. This behaviour can also be observed, if $\mathrm{Fe}$ is assumed to be fully ionized, and the ionization stage of He is varied (Fig. 4). Then Fe would diffuse in the same direction as $\mathrm{H}$, when mostly $\mathrm{He} \mathrm{I}$ is present in the plasma.

But in real stars such "anomalous" diffusion would rarely occur, as the ionization of hydrogen and helium leads to the development of convective regions, where mixing is much faster than diffusion. In these regions metals are, at least partially, ionized, too (Fig. 2). Therefore, in the diffusion-dominated radiative layers helium and metals almost never are in their lowest ionization stages, and thus the difference between $\bar{A}_{\mathrm{P}}$ and $A_{\mathrm{P}}^{\prime}$ remains small.

In some cases, however, the He abundances at the outer layers are reduced considerably by diffusion such that no convection develops due to $\mathrm{He}$ ionization. Then the diffusion of each He-ion should be treated separately. But taking into consideration that the diffusion velocity of He I is not determined very accurately and the He abundance in that case is very low, using the mean ionization degree of He may still yield sufficiently accurate results.

In summary, the diffusion of partly ionized elements in stars can in most relevant cases be determined by using one "effective" ion for each species carrying the mean ionization degree $\bar{\zeta}$. With this simplification the computation of the diffusion of partially ionized elements is not connected with larger computational time than using fully ionized particles and remains therefore feasible.

\section{Stellar and solar models}

For the calculation of the stellar models a descendant of the program by Kippenhahn et al. (1967) in the latest version described in Schlattl (2001) is used. In particular the opacities tables of Iglesias \& Rogers (1996) and the nuclear reaction rates given by Adelberger et al. (1998) are employed. While previously the equation-of-state tables of Rogers et al. (1996), known as OPAL- or ACTEX-EOS, has been implemented, now the analytic description of Irwin (2000) is applied. This step has become necessary, as the publicly accessible Rogers et al.'s (1996) tables do not provide quantities like the electron density or ionization degrees. Irwin (2000) equation of state (IRWINEOS) is a further development of the SIREFF-EOS, which itself was built from the EFF-EOS (Eggleton et al. 1973). While EFF could be solved during the stellar evolution program ("inline"), IRWIN is pretty slow, so that I produced spline tables on a pressure-temperature grid for different hydrogen and metal fractions. The metal distribution was chosen from Grevesse \& Noels (1993).

The chemical evolution is determined by solving simultaneously nuclear network and diffusion equation in a common scheme (Schlattl 1999), where convection is treated as a fast diffusive process. We follow the abundance changes of $\mathrm{H}$, ${ }^{3} \mathrm{He},{ }^{4} \mathrm{He},{ }^{12} \mathrm{C},{ }^{13} \mathrm{C},{ }^{14} \mathrm{~N},{ }^{15} \mathrm{~N},{ }^{16} \mathrm{O},{ }^{17} \mathrm{O},{ }^{20} \mathrm{Ne},{ }^{24} \mathrm{Mg}$, and ${ }^{56} \mathrm{Fe}$, which implies that $92 \%$ of the total metallic mass is considered.

\subsection{Solar models}

In my solar models the outer layers above an optical depth of 1000 have been taken from a 2D-hydrodynamical atmosphere model of Freytag et al. (1996), which is determining also the outer boundary condition of the interior solar model. These atmospheres extend down to regions, where the stratification is almost adiabatic, including the superadiabatic layers just below the surface. By this means improved p-mode frequencies could be obtained like with formerly applied 1D-atmospheres (Schlattl et al. 1997), and the models become almost independent of the convection theory applied. For the latter the description of Canuto \& Mazzitelli (1992) is used. As usual, the convection parameter $\alpha_{\mathrm{CM}}$, initial helium and metal content are adjusted to yield models with present solar luminosity, radius and metal fraction $Z / X$. In order to explore the effect of different diffusion approaches these solar constraints are reproduced with an accuracy better than $10^{-6}$. A summary of all solar models computed in this work is provided in Table 1.

In a first step models using the diffusion description of TBL containing OPAL- or IRWIN-EOS have been produced. Recently, an updated version of the OPAL-EOS has become available (denoted OPAL01, hereafter). The main differences compared to the version of 1996 (OPAL96) are the improvement of the activity expansion method for repulsive interactions (Rogers 2001), the inclusion of $\mathrm{HeH}^{+}$and $\mathrm{He}^{++}$ 
Table 1. Physics in solar models.

\begin{tabular}{lcccc}
\hline \hline \multirow{2}{*}{ Model } & \multirow{2}{*}{ EOS } & \multicolumn{3}{c}{ Effects included in diffusion } \\
& & non-ideal $\mathrm{e}^{-}$ & $P_{\text {rad }}$ & partly ionized \\
\hline OP1 & OPAL96 & - & - & - \\
OP2 & OPAL01 & - & - & - \\
IR1 & IRWIN & - & - & - \\
IR2 & IRWIN & $\mathrm{X}$ & - & - \\
IR3 & IRWIN & $\mathrm{X}$ & $\mathrm{X}$ & - \\
IR4 & IRWIN & $\mathrm{X}$ & $\mathrm{X}$ & $\mathrm{X}$ \\
\hline
\end{tabular}

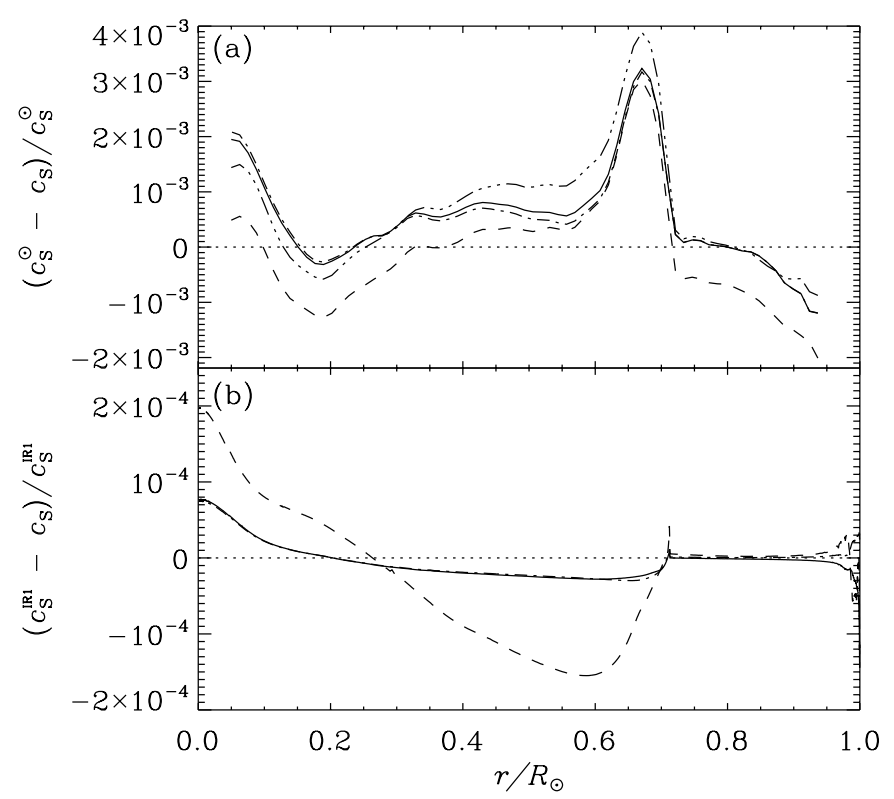

Fig. 5. a) Difference in the run of sound speed between the seismic model of Basu et al. (1997) and solar models OP1 (dashed line), OP2 (dash-dot-dot-dotted), IR1 (solid), and IR4 (dash-dotted), respectively. b) Sound speed of model IR2 (solid line), IR3 (dash dotted), and IR4 (dashed) compared to the reference model IR1.

in addition to $\mathrm{H}_{2}, \mathrm{H}_{2}^{+}$and $\mathrm{H}^{-}$, and a relativistic treatment of the electrons.

In Fig. 5a the differences of the sound speed between the seismic model of Basu et al. (1997) and models with OPAL96-, OPAL01-, and IRWIN-EOS, respectively, are shown. Interestingly, model OP2 containing the updated OPAL01-EOS provides a worse agreement on average with the solar sound-speed profile than model OP1 with the older OPAL96-EOS. The main reason is that the adiabatic index $\Gamma_{1}$ decreases when treating electrons relativistically (Bonanno et al. 2001). However, while the overall sound-speed profile has deteriorated, models with OPAL01-EOS provide a better agreement between the helioseismologically determined solar age and the meteoritic age of the solar system (Bonanno et al. 2002)

Model IR1 was computed with the IRWIN-EOS, where electrons are also treated relativistically and where $\mathrm{H}_{2}$ - and $\mathrm{H}_{2}^{+}-$ molecules are included. While in the central and convective region $\left(r>0.7 R_{\odot}\right.$ ), the sound speed of IR1 and OP2 are very similar, between $0.25<r / R_{\odot}<0.6$ IR 1 reproduces the

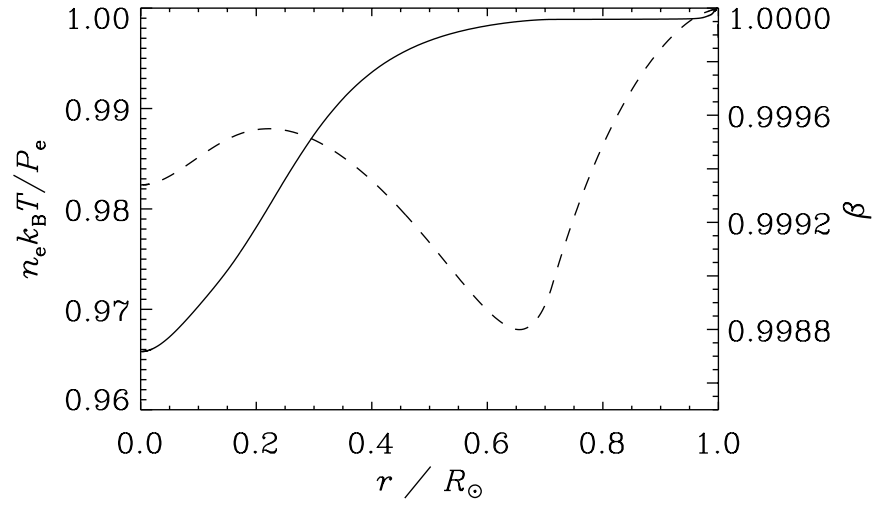

Fig. 6. The solid line shows the ratio between the electron pressure assuming an ideal gas $\left(n_{\mathrm{e}} k_{\mathrm{B}} T\right)$ and the actual electron pressure $P_{\mathrm{e}}$ in model IR1, while the dashed line represents $\beta$, the relative contribution of radiation to total pressure.

solar-sound speed profile better than model OP2. It is beyond the scope of this work to analyze what causes the difference between OPAL01- and IRWIN-EOS. Anyway, with the latter the solar sound-speed profile can be reproduced very well, and it thus provides an at least equally good description of stellar plasmas as the OPAL01-EOS.

Starting with model IR1 a series of solar models have been computed where subsequently non-standard effects have been added in the diffusion equation. The relevant physics included in each model is summarized in Table 1.

Since the electron degeneracy in the Sun is very small (Fig. 6), its implementation in the diffusion equation alters the sound speed at most only about $0.01 \%$ in the centre (Fig. 5b), where the degeneracy is highest.

An even smaller change is obtained by taking into account the radiation pressure which contributes at most about $0.1 \%$ at $r=0.65 R_{\odot}$ to the total pressure (Fig. 6). There a tiny change in the sound speed of about $10^{-5}$ can be observed (Fig. 5b), but overall it remains essentially invariant.

With the inclusion of partly ionized elements in the diffusion treatment the sound speed has increased by almost $0.02 \%$ at $r=0.6 R_{\odot}$, but decreased by about $0.01 \%$ in the centre compared to IR3. Taking into account that the sound speed of IR3 (and equally IR 1 ) has differed by about $0.08 \%$ from the seismic model for $0.3<r / R_{\odot}<0.65$, an improvement of about $25 \%$ could be achieved with model IR4.

In the present stage, however, the discrepancies between different input physics like equation of state are exceeding this value and thus the effect of partly ionized elements on diffusion appears to be negligible. But, including this effect reduces the uncertainty in solar models and thus helps to analyze the still existing shortcoming in model input physics.

Turcotte et al. (1998) has investigated the influence of a detailed treatment of the ionization stages in the diffusion equation, too. But in contrast to my results, they found a decrease of the sound speed when using partly instead of completely ionized elements. However, they followed a different strategy for computing solar models by using the same initial $Z / X$ ratio in the models under scrutiny. Furthermore, they failed to compare directly two models where the ions are assumed to 
Table 2. Characteristic quantities of solar models in this work. The indices i, s, c, and C.Z. denote initial, surface, core and bottom of convective envelope, respectively. $\alpha_{\mathrm{CM}}$ is the parameter of Canuto \& Mazzitelli's (1992) convection theory accounting for non-local effects, and $\Phi_{v}^{\mathrm{SK}}$ is the total flux of neutrinos produced in the ${ }^{8} \mathrm{~B}$-decay and the ${ }^{3} \mathrm{He}\left(\mathrm{p}, \mathrm{e}^{+} v_{\mathrm{e}}\right)^{4} \mathrm{He}$-reaction. In the last two columns the expected rates for the gallium $(\mathrm{Ga})$ and chlorine $(\mathrm{Cl})$ experiments are provided, where $1 \mathrm{SNU}$ (solar neutrino unit) corresponds to 1 event per $10^{36}$ target atoms and seconds.

\begin{tabular}{lccccccccccc}
\hline \hline Model & $Y_{\mathrm{i}}$ & $Z_{\mathrm{i}}$ & $\alpha_{\mathrm{CM}}$ & $Y_{\mathrm{s}}$ & $Z_{\mathrm{s}}$ & $\frac{R_{\mathrm{C} . \mathrm{.}}}{R_{\odot}}$ & $\frac{\rho_{\mathrm{c}}}{\mathrm{g} \mathrm{cm}^{-3}}$ & $\frac{T_{\mathrm{c}}}{10^{7} \mathrm{~K}}$ & $\frac{\Phi_{V}^{\mathrm{SK}}}{10^{6} \mathrm{~cm}^{-2} \mathrm{~s}^{-1}}$ & $\frac{\mathrm{Ga}}{\mathrm{SNU}}$ & $\frac{\mathrm{Cl}}{\mathrm{SNU}}$ \\
\hline OP1 & 0.27478 & 0.01992 & 0.97803 & 0.24429 & 0.01807 & 0.7131 & 152.01 & 1.5714 & 5.090 & 128.6 & 7.624 \\
OP2 & 0.27520 & 0.01987 & 1.0264 & 0.24509 & 0.01805 & 0.7132 & 152.02 & 1.5711 & 5.079 & 128.5 & 7.608 \\
IR1 & 0.27430 & 0.01987 & 1.0099 & 0.24450 & 0.01807 & 0.7123 & 151.87 & 1.5708 & 5.063 & 128.4 & 7.585 \\
IR2 & 0.27430 & 0.01987 & 1.0105 & 0.24448 & 0.01807 & 0.7124 & 151.92 & 1.5710 & 5.072 & 128.4 & 7.600 \\
IR3 & 0.27429 & 0.01987 & 1.0105 & 0.24449 & 0.01807 & 0.7124 & 151.93 & 1.5710 & 5.072 & 128.4 & 7.598 \\
IR4 & 0.27468 & 0.01996 & 1.0118 & 0.24482 & 0.01806 & 0.7125 & 151.98 & 1.5716 & 5.108 & 128.7 & 7.647 \\
\hline
\end{tabular}

Table 3. Abundance variations of solar models of this work. The central abundance of ${ }^{12} \mathrm{C}$ was in all models $0.7 \%$ of the initial one.

\begin{tabular}{lccrrrrr}
\hline \hline \multirow{2}{*}{ Model } & \multicolumn{4}{c}{ Surface (\%) } & \multicolumn{4}{c}{ Center (\%) } \\
& ${ }^{4} \mathrm{He}$ & ${ }^{12} \mathrm{C}$ & \multicolumn{1}{c}{$\mathrm{C} \mathrm{O}^{1} \mathrm{c}$} & \multicolumn{1}{c}{$\mathrm{C} \mathrm{He}$} & ${ }^{16} \mathrm{O}$ & $\mathrm{Z}$ \\
\hline OP1 & -11.1 & -10.3 & -10.1 & -10.1 & 133.1 & -2.2 & 8.0 \\
OP2 & -10.9 & -10.1 & -9.9 & -9.9 & 132.7 & -2.3 & 7.9 \\
IR1 & -10.9 & -10.1 & -9.9 & -9.9 & 133.3 & -2.3 & 7.9 \\
IR4 & -10.9 & -10.1 & -10.2 & -10.3 & 133.2 & -2.3 & 7.9 \\
\hline
\end{tabular}

be either fully or partly ionized. Instead they compared their model, which contained a detailed treatment of the ionization stages, with one where the diffusion velocities of all metals heavier than $\mathrm{O}$ are assumed to be equal to the velocity of completely ionized Fe. Both differences might contribute to the discrepancies between our results.

In Table 2 some characteristic quantities of the presented solar models are summarized. Only very small differences can be observed in all quantities. With the updated OPAL01- and IRWIN-EOS the core temperature has been reduced a little compared to model OP1 with the OPAL96-EOS. However, with the inclusion of partly ionized metals in the diffusion equation the temperature increases weakly, yielding a somewhat higher value for IR4 as for OP1, and thus slightly enhanced neutrino rates.

The abundance variations of ${ }^{4} \mathrm{He},{ }^{12} \mathrm{C}$, and ${ }^{16} \mathrm{O}$ at the solar surface and in the centre compared to their initial values are summarized in Table 3. Considering the detailed ionization stages of the metals in the diffusion scheme yield a higher reduction of ${ }^{16} \mathrm{O}$ and all metals on average, but the absolute changes remain small $(<0.4 \%)$. The biggest change can be observed for iron, which is about $2.5 \%$ stronger depleted in model IR4 than in IR3 (Fig. 7). This is in agreement with the result of Turcotte et al. (1998), but the overall change in the metal fraction of $0.4 \%$ is about a factor of 2 less than found by them. The reason is a smaller change of the diffusion velocity in my computations by using partly instead of completely ionized metals. In particular, the velocity of oxygen, which is the most abundant element among the metals, is increased only by about $5 \%$ below the convective envelope (Fig. 8), while

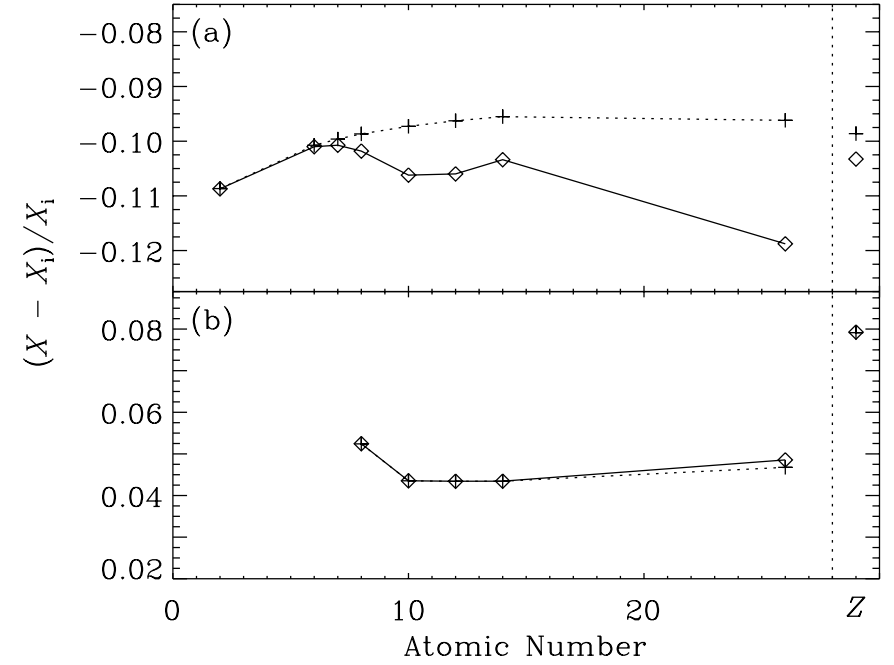

Fig. 7. a) The abundance changes at the surface between initial and solar model of sequence IR3 (dashed line) and IR4 (solid). On the right hand side the change in the total metallicity $Z$ is shown. b) As a) but in the centre. All elements lighter than $\mathrm{O}$ have been omitted, as their abundance is basically determined by the nuclear equilibrium of the $\mathrm{CNO}$ cycle.

Turcotte et al. (1998) found an about $10 \%$ higher value (see Fig. 13, therein).

The cause of this difference is difficult to disentangle, but it might be that the ionization fractions obtained from the IRWIN-EOS do not agree with the ones of the OPAL-EOS. The latter has been used by Turcotte et al. (1998). A further clue supporting this assessment is that the surface carbon abundance is almost unaltered in my models (Table 3), while Turcotte et al. (1998) found a depletion of $8.5 \%$ instead of $8 \%$. According to the IRWIN-EOS the ionization of $\mathrm{C}$ below the solar convective envelope is nearly complete (Fig. 2a), i.e., no change in the carbon depletion is expected, if the improved diffusion description is applied.

Independent of whether complete or partial ionization is assumed in the diffusion equation, the somewhat different diffusion velocities of each element cause a slight redistribution of metals, such that they are inconsistent with the metal composition assumed in the opacity tables. Turcotte et al. (1998) has 


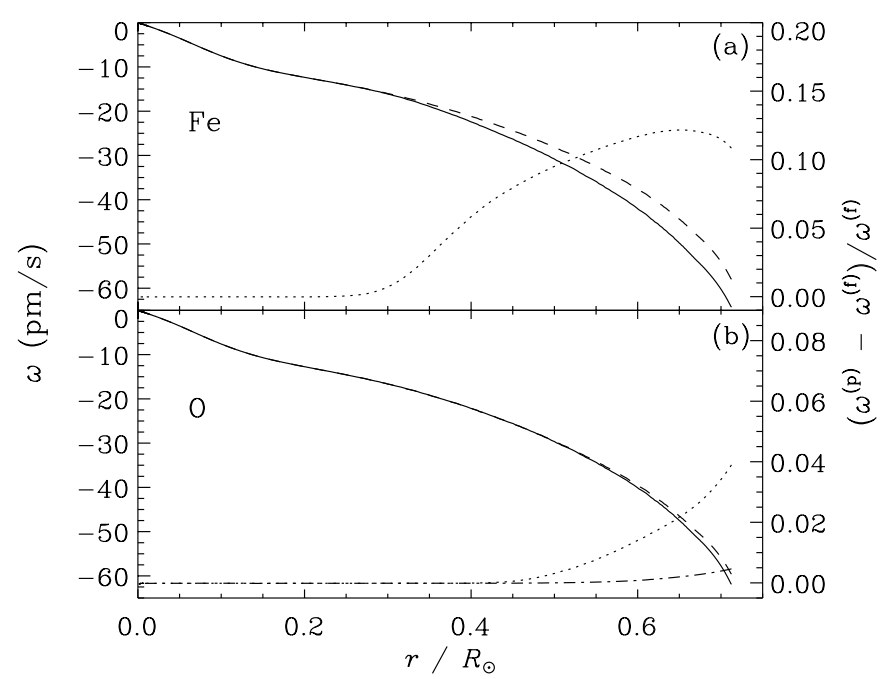

Fig. 8. a) The diffusion velocity of $\mathrm{Fe}$ in the Sun assuming fully (dashed line) resp. partly (solid) ionized elements and their relative difference (dashed line). b) Like a) but for $\mathrm{O}$. In addition the relative difference in the diffusion velocity of $\mathrm{C}$ between part and complete ionization are shown, too (dash-dotted line).

shown that the usage of monochromatic opacities may change the sound speed by about $0.1 \%$ compared to the Sun. They obtained this difference in a model with elaborate treatment of the ionization stages. However, as mentioned above, the metal distribution in their model was modified stronger compared to the initial one than in model IR4. So, using monochromatic opacities probably had a bigger effect on their model, than it would have on model IR4.

\subsection{Low-mass stars}

In addition to the solar models also models for low-mass metalpoor stars have been computed. The aim is to examine the influence of the improved diffusion scheme on their TO properties. Certainly the inclusion of radiative levitation can alter the models considerably (Richard et al. 2002), but it is nevertheless important to show how the more detailed treatment of ionization stages in the diffusion scheme modifies the stellar models.

Basically, the same input physics has been used as for the solar models, where for the equation of state Irwin's (2000) description has been applied. Since the 2D-atmospheres implemented in the solar models do not suffice in temperature, gravity and abundances for metal-poor stars, the empirical $T$ $\tau$-relation of Krishna Swamy (1966) has been included. For the convection Böhm-Vitense's (1958) mixing-length theory was employed with a mixing-length parameter of 1.77 , close to the calibrated value of a solar model containing the same physics. The metal distributions in the models were chosen to be $\alpha$-enhanced with $[\alpha / \mathrm{Fe}]=0.35$. Consistent opacity tables have been produced for this mixture (A. Weiss, private communication).

In Fig. 9 the evolution of $0.9,1.0$, and $1.1 M_{\odot}$ stars from the zero-age main sequence until the early-RGB are displayed in the HR-diagram. Their properties at the TO are summarized in Table 4. The inclusion of diffusion leads to smaller ages at

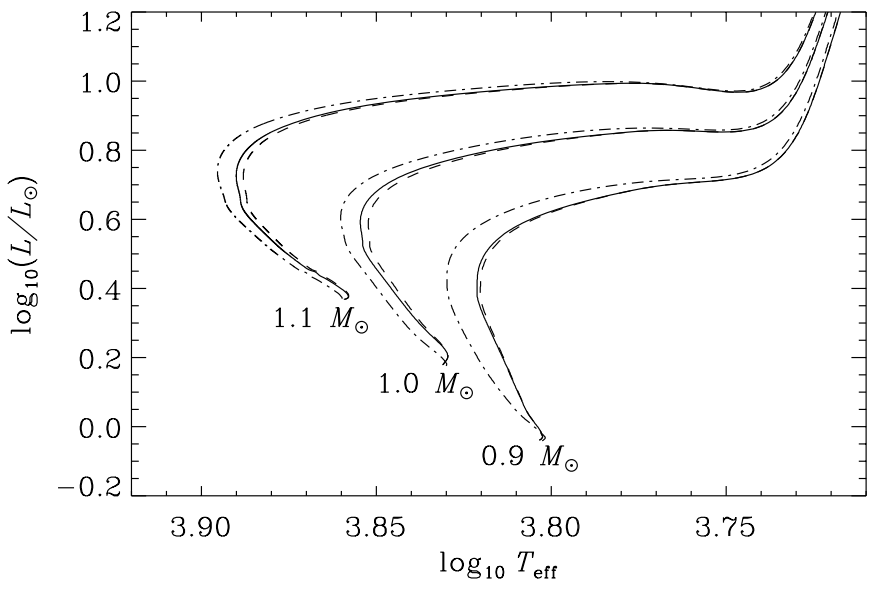

Fig. 9. The evolution of $0.9,1.0$, and $1.1 M_{\odot}$ stars with $[\mathrm{Fe} / \mathrm{H}]=-1.6$ ( $\alpha$-enhanced) treating all elements in the diffusion as partly (solid line) or fully ionized (dashed). For comparison the evolutions without diffusion are shown, too (dash-dotted line).

the TO, which has also been found by various authors. The effect of decreasing the age is more pronounced for smaller masses which have longer main-sequence lifetimes. There, diffusion is capable of decreasing the core hydrogen content considerably, the amount of which basically determines the time spent on the main sequence.

Strongly varying pressure and temperature gradients near the surface of these stars cause an almost total depletion of helium and metals at the surface near the TO phase. Thereby the degree of the helium depletion depends on two parameters, the extension of the convective envelope, which provides the reservoir for inward migrating atoms, and the time the star needs to reach the TO, i.e., the time diffusion can operate. The competing effects of these two parameters can be observed in the evaluated models. Since the mass of the convective envelope of the $1.0 M_{\odot}$ star is orders of magnitude smaller than in the $0.9 M_{\odot}$ star, helium is much more depleted in the former than in the latter case (Table 4). However, although the extension of the convective envelope is smallest in the $1.1 M_{\odot}$ star, more He remains on the surface than in the $1.0 M_{\odot}$ case; the reason for this is the smaller lifetime of the $1.1 M_{\odot}$ star on the main sequence.

The surface metal abundances follow the trend of He. However, their total depletion and distribution is strongly depending on the treatment of ions in the diffusion equation. Assuming complete ionization leads in general to smaller diffusion velocities (cf. Fig 3) and thus to a smaller decrease of the surface metal content. Already in this case the metal distribution may be altered up to a factor of 2 (Table 4). Taking into account the different ionization stages some metals may even totally disappear from the surface.

However, whether this also occurs in real stars is questionable, as with decreasing abundance and increasing effective temperature the influence of radiative levitation, which has been neglected here, becomes stronger. It would diminish the tendency of metals to settle in the stellar interior and might even lead to an enhancement of some elements on the surface (Richard et al. 2002). 
Table 4. Characteristic properties of low-mass stars at the TO with initial $Z=10^{-3}$ and $Y=0.24$, whereas an $\alpha$-enhanced mixture has been used with $[\alpha / \mathrm{Fe}]=0.35 . \Delta M_{\text {c.e. }}$ is the mass of the convective envelope. To show the changes in the abundances $\{x\} \equiv \log _{10}\left(x / x_{i}\right)$ has been defined, where the index $i$ denotes the initial value. The first row for each mass represents the no-diffusion case, while the second and third show the values considering fully and partly ionized elements, respectively, in the diffusion equation.

\begin{tabular}{rccccccccccccc}
\hline \hline$M_{\odot}$ & Age & $T_{\text {eff }}[\mathrm{K}]$ & $\frac{L}{L_{\odot}}$ & $\frac{\Delta M_{\text {c.e. }}}{M_{\odot}}$ & $\{Y\}$ & $\{Z\}$ & $\left\{\frac{X_{\mathrm{C}}}{Z}\right\}$ & $\left\{\frac{X_{\mathrm{N}}}{Z}\right\}$ & $\left\{\frac{X_{0}}{Z}\right\}$ & $\left\{\frac{X_{\mathrm{Ne}}}{Z}\right\}$ & $\left\{\frac{X_{\mathrm{Mg}}}{Z}\right\}$ & $\left\{\frac{X_{\mathrm{Si}}}{Z}\right\}$ & $\left\{\frac{X_{\mathrm{Fe}}}{Z}\right\}$ \\
\hline \multirow{2}{*}{0.9} & 7.75 & 6760 & 2.68 & $9.5 \times 10^{-6}$ & 0.0 & 0.0 & 0.00 & 0.00 & 0.00 & 0.00 & 0.00 & 0.00 & 0.00 \\
& 7.05 & 6613 & 2.40 & $7.7 \times 10^{-6}$ & -1.8 & -1.5 & -0.06 & -0.03 & -0.01 & 0.03 & 0.05 & 0.07 & 0.07 \\
& 7.07 & 6626 & 2.41 & $6.5 \times 10^{-6}$ & -1.9 & -2.1 & -0.22 & -0.03 & 0.09 & -0.05 & -0.42 & -0.88 & -1.34 \\
\hline \multirow{2}{*}{1.0} & 5.30 & 7248 & 4.03 & $6.0 \times 10^{-9}$ & 0.0 & 0.0 & 0.00 & 0.00 & 0.00 & 0.00 & 0.00 & 0.00 & 0.00 \\
& 5.03 & 7117 & 3.86 & $5.6 \times 10^{-9}$ & -3.3 & -2.0 & -0.32 & -0.17 & -0.06 & 0.11 & 0.21 & 0.29 & 0.34 \\
& 5.05 & 7154 & 3.89 & $4.0 \times 10^{-9}$ & -3.2 & -4.2 & -2.26 & -0.61 & 0.20 & -2.26 & $-\infty$ & $-\infty$ & $-\infty$ \\
\hline \multirow{3}{*}{1.1} & 3.63 & 7860 & 5.50 & $2.8 \times 10^{-9}$ & 0.0 & 0.0 & 0.00 & 0.00 & 0.00 & 0.00 & 0.00 & 0.00 & 0.00 \\
& 3.48 & 7727 & 5.29 & $1.0 \times 10^{-10}$ & -2.6 & -1.4 & -0.21 & -0.11 & 0.03 & 0.08 & 0.14 & 0.19 & 0.23 \\
& 3.50 & 7763 & 5.32 & $8.5 \times 10^{-11}$ & -2.5 & -2.8 & -2.12 & -0.59 & 0.18 & -0.32 & -5.34 & $-\infty$ & $-\infty$ \\
\hline
\end{tabular}

An additional source of uncertainty in these models is given by the strong deviation of the metal distribution from the initial one, being apparent in particular in models with detailed ionization treatment. With an assumed standard $\alpha$-enhanced mixture in the opacity tables, the opacities obtained are not valid any more at the TO, which may lead to different effective temperatures. However, Salaris et al. (1993) have demonstrated that the TO temperature of a $M=0.8 M_{\odot}$ star with a metallicity $Z=10^{-3}$ is determined basically by the opacities above about $12000 \mathrm{~K}$. Moreover, the opacities there are only sensitive to the contribution of $\mathrm{C}, \mathrm{N}, \mathrm{O}$, and $\mathrm{Ne}$ to the metals, whereas the detailed distribution of these elements is only of minor importance. Artificially decreasing their abundances, i.e., underestimating their contribution, yields too high effective temperatures.

In the models of this work, where full ionization is assumed, the contribution of $\mathrm{C}, \mathrm{N}, \mathrm{O}$, and Ne has been overestimated using standard $\alpha$-enhanced opacity tables (Fig. 10b), implying that the obtained effective temperature are actually too small. In way of contrast, just the opposite behaviour can be observed in the models where the ionization stages are considered in detail. The result is that the actual difference in effective temperature between models assuming either complete or partial ionization would be smaller as quoted in Table 4. From the work of Salaris et al. (1993), I conclude that the reduction would be about $10 \mathrm{~K}$.

Thus, when the redistributed metal abundances are taken into account in the opacities, the effective temperature of the $1.1 M_{\odot}$ star is increased only be about $20 \mathrm{~K}$ by treating ionization in the diffusion equation in detail. The effective temperature of the $0.9 M_{\odot}$ star remains almost unaltered. Probably, radiative levitation may slightly alter the effective temperature, but it is not expected that it alters the presented results by much more than $10 \mathrm{~K}$. In fact, Richard et al. (2002) have found an increase of about $10 \mathrm{~K}$ for a $0.8 M_{\odot}$ star when including radiative levitation and using monochromatic opacities. For the age determination of globular clusters the exact diffusion treatment is therefore only of minor importance, in particular, as the effect of the improved diffusion treatment is waning with decreasing TO mass, and thus increasing globular-cluster age.

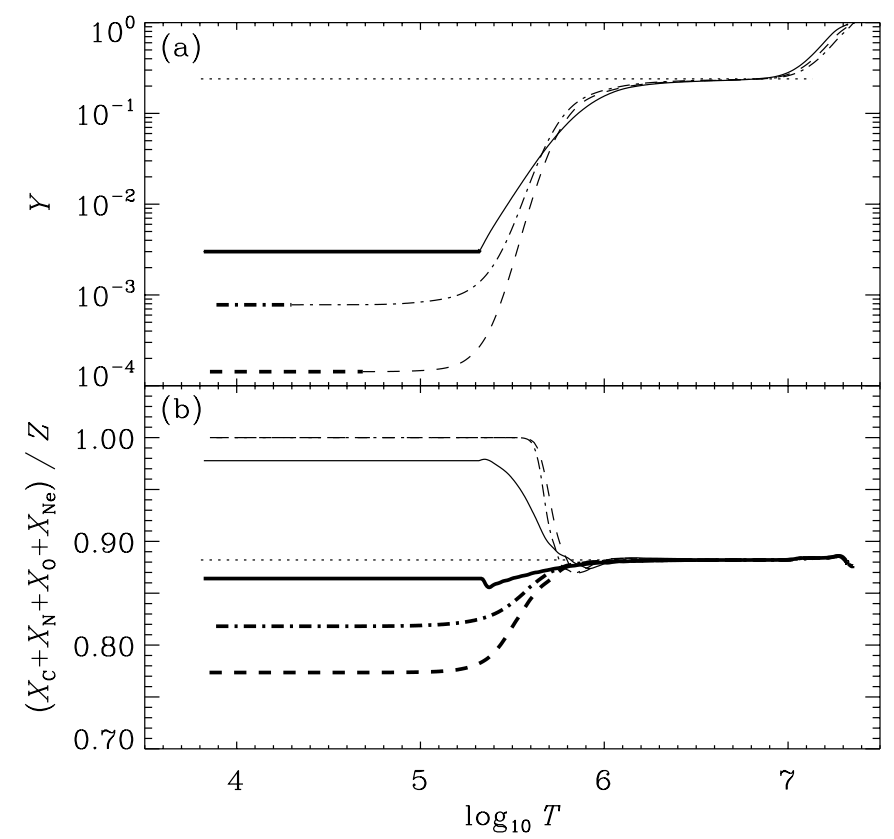

Fig. 10. a) The helium profile of a 0.9 (solid line), a 1.0 (dash-dotted), and a 1.1 (dashed) $M_{\odot}$ star at the TO including diffusion with elaborate treatment of the ionization stages. The thicker lines mark the envelope convective zones. b) The contribution of $\mathrm{C}, \mathrm{N}, \mathrm{O}$, and $\mathrm{Ne}$ to the total metallicity. The line styles agree with a), however, the thick lines correspond to models with complete ionization assumed. The dotted lines represent in both panels the initial values.

\section{Summary}

Starting from Burgers' equation for a multicomponent fluid the effect of partly ionized metals has been consistently included in the diffusion equation following the procedure of TBL. As an additional feature, electron degeneracy and the contribution of radiation to the total pressure has been taken into account. It has been shown, that for most stars it is sufficient to use one ion per element carrying the mean charge instead of computing diffusion for each ion separately. By this means, the elaborate treatment of partly ionized metals remains feasible. With increasing stellar mass, and thus decreasing stellar convective envelope, 
it may become necessary to consider, at least, all He-ions separately. However, since these stars are usually hotter, radiative levitation becomes important, which then should be included, too.

The effect of the improved diffusion treatment of partly ionized metals has been investigated in case of the Sun and the TO properties of low-mass metal-poor stars. In order to obtain the accurate ionization degrees of each metal species the equation of state of Irwin (2000) has been employed. The sound-speed profile in solar models obtained with this new EOS is in as good agreement with the helioseismic determined one as models with the updated OPAL01-EOS.

An improvement of up to $25 \%$ between $r=0.25 R_{\odot}$ and $r=0.65 R_{\odot}$ could be achieved by including the effect of partly ionization into the diffusion equation. However, this value is presently still exceeded by the uncertainties in other input physics like the EOS. Nevertheless, the changes between models treating metals fully and partly ionized have been found to be smaller than claimed by Turcotte et al. (1998). The origin of this discrepancy is not clear, but might be due to the use of different equations of state.

In low-mass metal-poor stars the improved diffusion description causes strong deviations in the surface metal distribution from the initial one. In addition, the depletion of metals is much stronger than in the case when full ionization is assumed. The consequent change in the effective temperature is at most about $40 \mathrm{~K}$ for a $1.1 M_{\odot}$ star diminishing with decreasing mass.

The influence of radiative levitation on the diffusive behaviour in particular of the metals has been neglected in this work. This may reduce the amount of depletion, and may further alter the metal distribution. Besides, since the effect of using partly instead of fully ionized metals in the diffusion equation is biggest for small convective envelopes, a small amount of additional mixing below the convective boundary may reduce the effect of diffusion strongly.

With the mass of the convective envelopes of metal-poor stars with $M>1 M_{\odot}$ being very small $\left(\lessgtr 10^{-8} M_{\odot}\right)$, also the amount of mass loss may strongly modify the surface abundances. However, mass-loss mechanisms are only very poorly understood. Therefore, first of all, precise stellar models are needed to disentangle the extent of mass loss and to determine the influence of rotation.

Acknowledgements. This work has been supported by a Marie Curie Fellowship of the European Community programme "Human Potential" under contract number HPMF-CT-2000-00951. I would like to thank M. Salaris for useful comments and inspiring discussions, and A. Weiss, who kindly provided splined versions of the opacity tables. In addition, I am grateful to A. W. Irwin for providing me his equation of state.

\section{Appendix A:}

The algebraic transformations of Eqs. (3)-(7) are toilsome but mathematically not very demanding. Therefore, only the basic intermediate steps and the final results are described here.
First, the r.h.s. of Eqs. (5) is converted, which consists according to Eq. (12) of two terms, where the first one, $\frac{\mathrm{d} \ln \beta P}{\mathrm{~d} r}$, is almost in its final shape

$\frac{\mathrm{d} \ln \beta P}{\mathrm{~d} r}=\frac{\mathrm{d} \ln P}{\mathrm{~d} r}+\frac{\mathrm{d} \ln \beta}{\mathrm{d} r}$.

Using Eq. (13), the remaining one can be written as

$$
\begin{array}{r}
\frac{\mathrm{d} \ln \left(C_{\mathrm{s}} / C\right)}{\mathrm{d} r}=\sum_{t \neq \mathrm{He}, e}\left(\delta_{\mathrm{ts}}-\frac{C_{\mathrm{t}}}{C}\right) \frac{\mathrm{d} \ln C_{\mathrm{t}}}{\mathrm{d} r}-\left(\delta_{\mathrm{s}, \mathrm{He}}-\frac{C_{\mathrm{He}}}{C}\right) \\
\times\left(\sum_{\mathrm{t} \neq \mathrm{He}} \frac{\zeta_{\mathrm{t}} \tilde{C}_{\mathrm{t}}}{\zeta_{\mathrm{He}} C_{\mathrm{He}}} \frac{\mathrm{d} \ln \tilde{C}_{\mathrm{t}}}{\mathrm{d} r}+\sum_{t \neq e} \frac{\zeta_{\mathrm{t}} C_{\mathrm{t}}}{\zeta_{\mathrm{He}} C_{\mathrm{He}}} \frac{\mathrm{d} \ln \zeta_{\mathrm{t}}}{\mathrm{d} r}\right),
\end{array}
$$

where "He" denotes either of the helium ions (not the neutral He!), and

$\tilde{C}_{\mathrm{s}}= \begin{cases}C_{\mathrm{s}}, & \text { for all ions } \\ \tilde{C}_{\mathrm{e}}, & \text { for electrons. }\end{cases}$

The 1.h.s. of the temperature equation (Eq. (3)) is given by Eq. (15).

The equations for current neutrality (Eq. (6)) and local mass conservation (Eq. (7)) become, using Eq. (10),

$\sum A_{\mathrm{s}} \tilde{C}_{\mathrm{s}} \omega_{\mathrm{s}}=0$

and

$\sum \zeta_{\mathrm{s}} \tilde{C}_{\mathrm{s}} \omega_{\mathrm{s}}=0$

where $A_{\mathrm{s}}$ is the mass of ion $\mathrm{s}$ in atomic units. The system of Eqs. (3)-(7) can now be written in the form

$$
\begin{aligned}
\frac{\beta P}{K_{0}}\left[\alpha_{i} \frac{\mathrm{d} \ln P}{\mathrm{~d} r}+\varphi_{i} \frac{\mathrm{d} \ln \beta}{\mathrm{d} r}\right. & +v_{i} \frac{\mathrm{d} \ln T}{\mathrm{~d} r}+\sum_{\substack{j=1 \\
j \neq 2}}^{S} \gamma_{i j} \frac{\mathrm{d} \ln \tilde{C}_{j}}{\mathrm{~d} r} \\
& \left.+\sum_{\substack{j=1 \\
j \neq S}}^{S} \lambda_{i j} \frac{\mathrm{d} \ln \zeta_{j}}{\mathrm{~d} r}\right]=\sum_{j=1}^{2 S+2} \Delta_{i j} W_{j},
\end{aligned}
$$

where $\mathrm{He}$ is supposed to be species No. 2 and the electrons species No. $S$. The quantities $K_{0}, \boldsymbol{\alpha}, \boldsymbol{v}, \gamma, \Delta$, and $\boldsymbol{W}$ are defined as in TBL with the following exceptions:

$$
\begin{aligned}
& \text { 1. } \gamma_{i j}=\frac{C_{i}}{C}\left[\left(1-\delta_{j S}\right)\left(\delta_{i j}-\frac{C_{j}}{C}\right)-\left(\delta_{i 2}-\frac{C_{2}}{C}\right) \frac{\zeta_{j} \tilde{C}_{j}}{\zeta_{2} C_{2}}\right] \text { for } \\
& i=1, \ldots, S \text { (cf. Eq. }(\text { A. } 1)) \\
& \text { 2. } \Delta_{i j}= \begin{cases}\left.\left.A_{j} \tilde{C}_{j} \text { for } j=1, \ldots, S ; i=2 S+1 \text { (cf. Eq. (A. } 2\right)\right) \\
\left.\left.\zeta_{j} \tilde{C}_{j} \text { for } j=1, \ldots, S ; i=2 S+2 \text { (cf. Eq. (A. } 3\right)\right)\end{cases} \\
& \text { 3. } \Delta_{i j}= \begin{cases}\zeta_{j} \tilde{C}_{j} & \text { for } j=2 S+1 ; i=1, \ldots, S \\
-A_{j} \tilde{C}_{j} \text { for } j=2 S+2 ; i=1, \ldots, S\end{cases} \\
& \text { 4. } W_{j}= \begin{cases}K_{0}^{-1} \tilde{n}_{\mathrm{e}} e E & \text { for } j=2 S+1 \\
K_{0}^{-1} \beta \tilde{n}_{\mathrm{e}} m_{0} g \text { for } j=2 S+2,\end{cases}
\end{aligned}
$$

where $m_{0}$ is the atomic mass unit and $K_{0}=\frac{3}{4} \sqrt{\frac{m_{0}}{2 \pi}} k_{\mathrm{B}}^{5 / 2} \mathrm{e}^{-4}$. Finally, the new coefficients $\varphi$ and $\lambda$ are defined (see Eq. (A.1)) as

$\varphi_{i}=\frac{C_{i}}{C}-\frac{A_{i} \tilde{C}_{i}}{\sum_{i} A_{i} \tilde{C}_{i}} \quad$ for $i=1, \ldots, S$ 
and

$\lambda_{i j}= \begin{cases}-\frac{C_{i}}{C}\left(\delta_{i 2}-\frac{C_{2}}{C}\right) \frac{\zeta_{j} C_{j}}{\zeta_{2} C_{2}} & \text { for } i=1, \ldots, S-1 \\ 0 & \text { for } i=S, \ldots, 2 S+2 .\end{cases}$

To account for the partly ionized metals in the particle interaction, TBL's $Z_{\mathrm{s}}$ has to be substituted by $\zeta_{\mathrm{s}}$ in the cross section $\sigma_{\text {st }}$ (TBL's Eq. (8)), and in the Coulomb logarithm $\ln \Lambda_{\text {st }}$ (TBL's Eq. (9)).

\section{References}

Adelberger, E. G., Austin, S. M., Bahcall, J. N., et al. 1998, Rev. Mod. Phys., 70, 1265

Babel, J., \& Michaud, G. 1991, A\&A, 248, 155

Bahcall, J. N., Basu, S., \& Pinsonneault, M. H. 1998, Phys. Lett. B, 433,1

Bahcall, J. N., \& Pinsonneault, M. H. 1992, Rev. Mod. Phys., 64, 885

Basu, S., Chaplin, W. J., Christensen-Dalsgaard, J., et al. 1997, MNRAS, 292, 243

Böhm-Vitense, E. 1958, ZAp, 46, 108

Bonanno, A., Murabito, A. L., \& Paternò, L. 2001, A\&A, 375, 1062

Bonanno, A., Schlattl, H., \& Paternò, L. 2002, A\&A, 390, 1115

Burgers, J. M. 1969, Flow Equations for Composite Gases (New York, London: Academic Press)

Canuto, M., \& Mazzitelli, I. 1992, ApJ, 389, 724

Castellani, V., Ciacio, F., degl'Innocenti, S., \& Fiorentini, G. 1997, A\&A, 322, 801

Chaboyer, B., Fenton, W. H., Nelan, J. E., Patnaude, D. J., \& Simon, F. E. 2001, ApJ, 562, 521

Eggleton, P. P., Faulkner, J., \& Flannery, B. P. 1973, A\&A, 23, 325
Freytag, B., Ludwig, H.-G., \& Steffen, M. 1996, A\&A, 313, 497

Gonzalez, J.-F., LeBlanc, F., Artru, M.-C., \& Michaud, G. 1995, A\&A, 297, 223

Grevesse, N., \& Noels, A. 1993, Phys. Scripta, T47, 133

Iglesias, C. A., \& Rogers, F. J. 1996, ApJ, 464, 943

Irwin, A. W. 2000, GNU public license, FORTRAN routines available at ftp://astroftp. phys.uvic.ca/pub/irwin/eos/code

Kippenhahn, R., Weigert, A., \& Hofmeister, E. 1967, Methods in Computational Physics, vol. 7, Methods for Calculating Stellar Evolution (New York: Academic Press), 129

Krishna Swamy, K. S. 1966, ApJ, 145

Michaud, G., Martel, A., \& Ratel, A. 1978, ApJ, 226, 483

Ramírez, S. V., \& Cohen, J. G. 2002, AJ, 123, 3277

Ramírez, S. V., Cohen, J. G., Buss, J., \& Briley, M. M. 2001, AJ, 122, 1429

Richard, O., Michaud, G., Richer, J., et al. 2002, ApJ, 568, 979

Richard, O., Vauclair, S., Charbonnel, C., \& Dziembowski, W. A. 1996, A\&A, 312, 1000

Richer, J., Michaud, G., \& Turcotte, S. 2000, ApJ, 529, 338

Rogers, F. J. 2001, Contrib. Plasma Phys., 41, 179

Rogers, F. J., Swenson, F. J., \& Iglesias, C. A. 1996, ApJ, 456, 902

Salaris, M., Chieffi, A., \& Straniero, O. 1993, ApJ, 414, 580

Salaris, M., Groenewegen, M. A. T., \& Weiss, A. 2000, A\&A, 355, 299

Schlattl, H. 1999, Ph.D. Thesis, Technical University Munich

Schlattl, H. 2001, Phys. Rev. D, 64, 013009

Schlattl, H., Weiss, A., \& Ludwig, H.-G. 1997, A\&A, 322, 646

Thoul, A. A., Bahcall, J. N., \& Loeb, A. 1994, ApJ, 421, 828

Turcotte, S., Richer, J., Michaud, G., Iglesias, C. A., \& Rogers, F. J. 1998, ApJ, 504, 539

Weiss, A., \& Schlattl, H. 2000, A\&AS, 144, 487 